\title{
Effects of sodium houttuyfonate on transcriptome of Pseudomonas aeruginosa
}

\author{
Yeye Zhao ${ }^{1}$, Yuanqing $\mathrm{Si}^{1}$, Longfei Mei ${ }^{1}$, Jiadi Wu' ${ }^{1}$, Jing Shao ${ }^{1}$, Changzhong Wang ${ }^{1}$ and Daqiang $\mathrm{Wu}^{1}$, $^{*}$
}

\begin{abstract}
Objectives: The purpose of this experiment is to analyze the changes of transcriptome in Pseudomonas aeruginosa under the action of sodium houttuyfonate (SH) to reveal the possible mechanism of SH inhibiting P. aeruginosa. We analyzed these data in order to compare the transcriptomic differences of P. aeruginosa in SH treatment and blank control groups.

Data description: In this project, RNA-seq of BGISEQ-500 platform was used to sequence the transcriptome of $P$. aeruginosa, and sequencing data of 8 samples of $P$. aeruginosa are generated as follows: $\mathrm{SH}$ treatment $(\mathrm{SH} 1, \mathrm{SH} 2, \mathrm{SH} 3$, $\mathrm{SH} 4$ ), negative control (Control 1, Control 2, Control 3, Control 4). Quality control is carried out on raw reads to determine whether the sequencing data is suitable for subsequent analysis. Totally $170.53 \mathrm{MB}$ of transcriptome sequencing data is obtained. Then the filtered clean reads are aligned and compared to the reference genome to proceed second quality control. After completion, 5938 genes are assembled from sequencing data. Further quantitative analysis of genes and screening of differentially expressed genes based on gene expression level reveals that there are 2047 significantly differentially expressed genes under SH treatment, including 368 up-regulated genes and 1679 downregulated genes.
\end{abstract}

Keywords: RNA-seq, Pseudomonas aeruginosa, Sodium houttuyfonate, Transcriptome

\section{Objective}

Pseudomonas aeruginosa is a gram-negative bacterium, which can produce endotoxin, exotoxin, proteolytic enzyme and other substances and infect human and other organisms [1,2]. At present, macrolide and aminoglycoside antibiotics are commonly used for curing the clinical infection of $P$. aeruginosa. However, with the emergence of drug resistance, $P$. aeruginosa are difficult to treat by common antibiotics. Thus, we are seeking for effective antimicrobial agents from traditional Chinese medicine to treat infection of $P$. aeruginosa. Previously, our research group have proved that sodium houttuyfonate $(\mathrm{SH})$ can effectively inhibit the $P$. aeruginosa [3, 4]. Here, our aim is to investigate possible antimicrobial mechanism of $\mathrm{SH}$ by comparing the transcriptomic differences between SH drug and blank control groups.

\footnotetext{
*Correspondence: daqwu@126.com

2 Key Laboratory of Xin'An Medicine, Ministry of Education, Anhui University of Chinese Medicine, Hefei 230038, China

Full list of author information is available at the end of the article
}

The assemble transcriptome contains thousands of transcripts. Thus this study provides transcriptomic comparison between $\mathrm{SH}$ medication group and blank control group rather than comparisons of expression of several certain genes such as $\operatorname{alg} D$, algR, lasI, phzM, las $A$ and $b d l A$, in previous studies [4-6]. The difference of these transcriptome can be used as the basis for studying gene expression changes in $\mathrm{SH}$ treatment and control groups.

\section{Data description}

We cultured $P$. aeruginosa under two conditions, with 4 biological replications which were cultured independently under each condition:

1. ATCC 27853 was inoculated into LB liquid medium and cultured overnight at $37{ }^{\circ} \mathrm{C}$. The culture were centrifuged for $1 \mathrm{~min}$ at $12,000 \mathrm{r} / \mathrm{min}$, and pour out the supernatant, diluted with sterile water to 0.5 Maxwell colorimetric tube, and diluted to $10^{7}$ times for later use. The $\mathrm{SH}$ was prepared according to our previous research [6]. The prepared $\mathrm{SH}$ samples in 
LB medium with 1 MIC (minimum inhibitory concentration) SH of $512 \mu \mathrm{g} / \mathrm{ml}$ were cultured for $24 \mathrm{~h}$ at $37^{\circ} \mathrm{C}$ until $\mathrm{OD}_{600}$ was $0.6-0.8$, and collected by centrifugation of $1 \mathrm{~min}$ at $12,000 \mathrm{r} / \mathrm{min}$, and then rinsed with sterile water for 3 times. Then we placed the collected bacterial samples in the centrifuge tube, sealed it with sealing film, and sent the sample for RNA-seq with dry ice.

2. The samples of blank control were collected similarly as $\mathrm{SH}$ treatment samples except without drug treatment.

Totally $170.53 \mathrm{MB}$ of transcriptome sequencing data is obtained after RNA-seq applying BGISEQ-500 platform. The original data of sequencing includes reads with low quality, linker contamination and high content of unknown base $\mathrm{N}$ are removed before data analysis to ensure the reliability of the results. This project used SOAPnuke [7], a filtering software independently developed by Huada Corporation, to make statistics and trimmomatic [8] to filter. Firstly, readers including connectors are removed. Then the reads with unknown base $\mathrm{N}$ content more than $5 \%$ are wiped off. Finally, the low-quality reads are removed (we define reads with a mass value of less than 10 and a proportion of more than $20 \%$ of the total number of bases in the reads as low-quality reads). The filtered "Clean Reads" are saved in FASTQ format. The file format corresponding to each sample is FASTQ format (Table 1).

The original sequencing sequence data (fastq file), including reference genome information, can be obtained on NCBI. After the quality control of the original data, we used Bowtie2 [9] to compare clean reads to the reference gene sequence (Table S1), and then RSEM [10] was used to calculate the expression levels of genes and transcripts. After completion, totally 5938 genes are assembled from sequencing data.

In order to reflect the correlation of gene expression between samples, Pearson correlation coefficients of all gene expression amounts between every samples are calculated, and expression amount distribution analysis are performed. The obtained results are shown in Fig. S1A [13]. According to the gene expression level of each sample, a total of 2047 differentially expressed genes are detected by the threshold of fold changes $>2$, $Q$ value $<0.001$, including 368 up-regulated genes and 1679 down-regulated genes. The results are shown in volcanic map of Fig. S1B [13].

\section{Limitations}

The limitations of this data is that there is not a gradient comparison under multiple different concentrations of $\mathrm{SH}$, and the transcriptome expression of $P$. aeruginosa under $24 \mathrm{~h}$ of culture is selected in this study, which may make the results inconsistent with other research results. In addition, in our previous studies $[4,5]$, the qRT-PCR results showed that genes of $\operatorname{las} A, \operatorname{alg} D, \operatorname{alg} R$ are down regulated by $\mathrm{SH}$ treatment in $P$. aeruginosa. However, these genes are found to be below the detection threshold in this study. This may be due to the different technologies used in determination of the gene expression.

Table 1 Overview of transcriptome data files

\begin{tabular}{|c|c|c|c|}
\hline Label & Name of data file/data set & $\begin{array}{l}\text { File types (file } \\
\text { extension) }\end{array}$ & Data repository and identifier (DOI or accession number) \\
\hline Control1 & SRR9031329_1.fq & fastq & https://www.ncbi.nlm.nih.gov/sra/SRX5808580[accn] \\
\hline Control2 & SRR9031328_1.fq & fastq & https://www.ncbi.nlm.nih.gov/sra/SRX5808581 [accn] \\
\hline Control3 & SRR9031319_1.fq & fastq & https://www.ncbi.nlm.nih.gov/sra/SRX5808590[accn] \\
\hline Control4 & SRR9031318_1.fq & fastq & https://www.ncbi.nlm.nih.gov/sra/SRX5808591 [accn] \\
\hline $\mathrm{SH} 1$ & SRR9031323_1.fq & fastq & https://www.ncbi.nlm.nih.gov/sra/SRX5808586[accn] \\
\hline $\mathrm{SH} 2$ & SRR9031322_1.fq & fastq & https://www.ncbi.nlm.nih.gov/sra/SRX5808587[accn] \\
\hline $\mathrm{SH} 3$ & SRR9031321_1.fq & fastq & https://www.ncbi.nlm.nih.gov/sra/SRX5808588[accn] \\
\hline $\mathrm{SH} 4$ & SRR9031320_1.fq & fastq & https://www.ncbi.nlm.nih.gov/sra/SRX5808589[accn] \\
\hline Gene expression data & $\begin{array}{l}\text { GSE133428_All_samples.Gene- } \\
\text { Expression.FPKM }\end{array}$ & txt.gz & https://www.ncbi.nlm.nih.gov/geo/query/acc.cgi?acc=GSE133428 \\
\hline Table S1 & SH transcriptome-S1.xlsx & $x \mid s x$ & https://doi.org/10.6084/m9.figshare.8241410.v1 \\
\hline Figure S1 & Figure S1 & .tif & https://doi.org/10.6084/m9.figshare.8241410.v1 \\
\hline
\end{tabular}

The raw RNA-Seq data (.fastq files) are available for download on the SRA [11]. The gene expression data (.txt.gz) are available on the GEO [12]. The additional files including Table S1 and Figure S1 can obtained on Figshare [13] 


\section{Abbreviations}

RNA-seq: ribonucleic acid sequencing; SH: sodium houttuyfonate; qRT-PCR: quantitative reverse transcription polymerase chain reaction.

\section{Acknowledgements}

We thank Professor Huang Xianqing of Shanghai Jiaotong University for providing $P$. aeruginosa strain and the sequencing facilities of Huada Gene Cloud Platform for the preparation and sequencing of the library.

\section{Authors' contributions}

DQW, JS and CZW designed and funded the experiment. YZ, YQS, LFM and JDW conducted experiments, and wrote the manuscript. All authors read and approved the final manuscript.

\section{Funding}

The research is funded by National Natural Science Foundation of China (Grant No. 81503115) and the Discipline Construction Key Program of Anhui University of Chinese Medicine (DC18100042). The Funding is used to cover laboratory expenses, sample preparation and sequencing. These funding agencies have no role in research design, data collection, analysis of results, or manuscript writing.

\section{Availability of data materials}

The data described in this data description can be freely and publicly accessed on the sequence reading file (SRA) with the item number of http://identifier s.org/ncbi/insdc.sra:SRP197195 [11] and the gene expression omnibus (GEO) with the item number of https://www.ncbi.nlm.nih.gov/geo/query/acc. cgi?acc=GSE133428 [12]. We have used these data to conduct a large amount of additional analysis, and these results can be obtained from https://figshare. com/articles/SH_transcriptome-S1_xIsx/8241410 [13] and the corresponding authors according to reasonable requirements.

\section{Ethics approval and consent to participate} Not applicable.

\section{Consent for publication}

Not applicable.

\section{Competing interests}

The authors declare that they have no competing interests.

\section{Author details}

${ }^{1}$ Department of Pathogenic Biology and Immunology, College of Integrated Chinese and Western Medicine, Anhui University of Chinese Medicine, Hefei 230012, China. ${ }^{2}$ Key Laboratory of Xin'An Medicine, Ministry of Education, Anhui University of Chinese Medicine, Hefei 230038, China.

Received: 9 June 2019 Accepted: 12 October 2019

Published online: 22 October 2019

\section{References}

1. Denning GM, lyer SS, Reszka KJ, et al. Phenazine-1-carboxylic acid, a secondary metabolite of Pseudomonas aeruginosa, alters expression of immunomodulatory proteins by human airway epithelial cells. Am J Physiol Lung Cell Mol Physiol. 2003;285(3):584-92. https://doi. org/10.1152/ajplung.00086.2003.

2. Cui Q, Li F, Xing W, et al. Differential expression of two phenazineproducing loci mediated by deficiency of the global regulator rsmA in Psedomonas aeruginosa PAO1. Wei Sheng Wu Xue Bao. 2012;52:1326-34.

3. Cheng HJ, Wang CZ, Lu WB, et al. Effects of houttuyfonate sodium on eliminating adhesion of Psedomonas aeruginosa and forming biofilms. Zhongguo Zhong Yao Za Zhi. 2012;37:3297-301.

4. Wu DQ, Cheng H, Huang W, Duan Q. Sodium houttuyfonate in vitro inhibits the biofilm formation and alginate biosynthesis gene expression of Pseudomonas aeruginosa clinical strain. Exp Ther Med. 2015;10:753-8. https://doi.org/10.3892/etm.2015.2562.

5. Wu D, Huang W, Duan Q, Li F, Cheng H. Sodium houttuyfonate affects production of $\mathrm{N}$-acyl homoserine lactone and quorum sensing-regulated genes expression in Pseudomonas aeruginosa. Front microbiol. 2014:5:635. https://doi.org/10.3389/fmicb.2014.00635.

6. Wang Tianming, Huang Weifeng, Duan Qiangjun, Wang Jian, Cheng Huijuan, Shao Jing, Li Fang, Daqiang Wu. Sodium houttuyfonate in vitro inhibits biofilm dispersion and expression of bdlA in Pseudomonas aeruginosa. Mol Biol Rep. 2019;46(1):471-7. https://doi.org/10.1007/s11033-018-4497-9.

7. Chen Y, Chen Y, Shi C, et al. SOAPnuke: a MapReduce accelerationsupported software for integrated quality control and preprocessing of high-throughput sequencing data. Gigascience. 2018;7(1):1-6. https:// doi.org/10.1093/gigascience/gix120.

8. Bolger AM, Lohse M, Usadel B. Trimmomatic: a flexible trimmer for illumina sequence data. Bioinformatics. 2014;30(15):2114-20. https://doi. org/10.1093/bioinformatics/btu170.

9. Langmead B, Salzberg SL. Fast gapped-read alignment with Bowtie 2. Nat Methods. 2012;9(4):357-9. https://doi.org/10.1038/nmeth.1923.

10. Dewey CN, Bo L. RSEM: accurate transcript quantification from RNA-Seq data with or without a reference genome. BMC Bioinform. 2011;12(1):323. https://doi.org/10.1186/1471-2105-12-323.

11. Wu D, Zhao Y. RNA-Seq of Pseudomonas aeruginosa. Sequence Read Archive. 2019. http://identifiers.org/ncbi/insdc.sra:SRP197195. Accessed 11 Jun 2019.

12. Wu D, Zhao Y. Effect of sodium houttuyfonate on transcriptome of Pseudomonas aeruginosa. https://www.ncbi.nlm.nih.gov/geo/query/acc. cgi?acc=GSE133428. Accessed 28 Jun 2019.

13. Wu D, Zhao Y. SH transcriptome-S1.xlsx. Figshare. 2019. https://doi. org/10.6084/m9.figshare.8241410.v1.

\section{Publisher's Note}

Springer Nature remains neutral with regard to jurisdictional claims in published maps and institutional affiliations.

Ready to submit your research? Choose BMC and benefit from:

- fast, convenient online submission

- thorough peer review by experienced researchers in your field

- rapid publication on acceptance

- support for research data, including large and complex data types

- gold Open Access which fosters wider collaboration and increased citations

- maximum visibility for your research: over $100 \mathrm{M}$ website views per year

At BMC, research is always in progress.

Learn more biomedcentral.com/submissions 